\title{
O Cuidado de Enfermagem a criança com transtorno do espectro autista: um desafio no cotidiano
}

\author{
Nursing Care for children with autism spectrum disorder: a daily challenge \\ Cuidado de enfermería para niños con trastorno del espectro autista: un desafío diario
}

Lara de Brito Ribas ${ }^{1 *}$, Manoela Alves ${ }^{2}$

Como citar esse artigo. Ribas, LB; Alves, M. O Cuidado de Enfermagem a criança com transtorno do espectro autista: um desafio no cotidiano. Revista Pró-UniverSUS. 2020 Jan./Jun.; 11 (1): $74-79$

\begin{abstract}
Resumo
O trabalho trata do cuidado de enfermagem a criança autista. Tendo por objetivos: descrever o cuidado de enfermagem a criança autista e analisar o cuidado de enfermagem a criança autista. Trata-se de uma revisão bibliográfica, através de estudos científicos, nas bases de dados mais relevantes como: Scielo (Scientific Electronic Library Online) e BIREME. Foram encontrados inicialmente 1.278 artigos que envolviam o tema de autismo. Após aplicar os DECS (cuidados de enfermagem, transtorno do espectro autista, autismo), filtrou-se 8 artigos que abordavam a temática, no idioma Português e no período de 2014-2019. Após avaliação dos títulos, encontraram-se o total de 6 artigos, com a leitura minuciosa dos artigos foram excluídos o total de 3 artigos, resultando em somente 3 artigos que abordavam a temática dos cuidados de enfermagem ao autismo infantil. Os dados demonstram um grande déficit sobre a temática. A prática assistencial do enfermeiro frente à criança autista, ainda é um tabu para muitos profissionais, o que decorre da falta de estudos científicos que abordem o assunto. Conclui-se, que há a necessidade de que a temática seja ministrada na graduação, a fim de que sejam produzidos estudos que capacitem os profissionais enfermeiros, proporcionando uma assistência qualificada.
\end{abstract}

Palavras-chave: Autismo, Criança, Cuidado de enfermagem.

\begin{abstract}
This paper deals with nursing care for autistic children. Having as objectives: to describe the nursing care of the autistic child and to analyze the nursing care of the autistic child. This is a bibliographic review, through scientific studies, in the most relevant databases such as: Scielo (Scientific Electronic Library Online) and BIREME. We initially found 1,278 articles involving the topic of autism. After applying the DECS (nursing care, autism spectrum disorder, autism), we filtered 8 articles that addressed the theme, in Portuguese and in the period 2014-2019. After evaluating the titles, a total of 6 articles were found, with a thorough reading of the articles, the total of 3 articles were excluded, resulting in only 3 articles that addressed the theme of nursing care for childhood autism. Data show a large deficit. about the theme. Nursing care practice towards autistic children is still a taboo for many professionals, which results from the lack of scientific studies that address the subject. It is concluded that there is a need for the theme to be taught at undergraduate level, so that studies are produced that enable nursing professionals, providing qualified assistance.
\end{abstract}

Keywords: Autism, Kid, Nursing Care.

\section{Resumen}

Este artículo trata sobre el cuidado de enfermería para niños autistas. Tener como objetivos: describir los cuidados de enfermería del niño autista y analizar los cuidados de enfermería del niño autista. Esta es una revisión bibliográfica, a través de estudios científicos, en las bases de datos más relevantes, tales como: Scielo (Scientific Electronic Library Online) y BIREME. Inicialmente encontramos 1.278 artículos relacionados con el tema del autismo. Después de aplicar el DECS (cuidados de enfermería, trastorno del espectro autista, autismo), filtramos 8 artículos que abordaron el tema, en portugués y en el período 2014-2019. Después de evaluar los títulos, se encontraron un total de 6 artículos, con una lectura exhaustiva de los artículos, se excluyeron el total de 3 artículos, lo que resultó en solo 3 artículos que abordaron el tema del cuidado de enfermería para el autismo infantil. Los datos muestran un gran déficit. sobre el tema La práctica del cuidado de enfermería para niños autistas sigue siendo un tabú para muchos profesionales, como resultado de la falta de estudios científicos que aborden el tema. Se concluye que es necesario que el tema se enseñe a nivel de pregrado, de modo que se produzcan estudios que permitan a los profesionales de enfermería, brindando asistencia calificada.

Palabras clave: Autismo, Cuidados de Enfermaría, Niño

Afiliação dos autores: 1. Discentes de Enfermagem da Universidade de Vassouras. Vassouras - Rio de Janeiro - Brasil. Email: rbritolara@gmail.com ORCID: https://orcid.org/00000002-9660-804X

2. Docente na Universidade de Vassouras. Vassouras - Rio de Janeiro - Brasil Email: alves.manoela@gmail.com ORCID: : https://orcid.org/0000-0003-4239-9577

* Email de correspondencia: rbritolara@gmail.com 


\section{Introdução}

A enfermagem iniciou sua prática no campo psiquiátrico a partir da assistência manicomial, associada ao suprimento das necessidades que a equipe médica encontrava para efetuar o tratamento de seus pacientes.

Sabe-se que por décadas, o paciente psiquiátrico foi tratado como um ser sem perspectiva de melhoras. Nesta época, a assistência de enfermagem à esse paciente era restrita a determinadas ações, tais como: medicação e sedação, cautela através da guarda constante desses pacientes no âmbito manicomial e por fim, medidas de penalidades, que envolviam as mais rudes torturas ${ }^{1}$.

Com o passar dos anos, foi instituído no Brasil o movimento de reforma psiquiátrica. O movimento deu início no final da década de 70 e tinha por objetivo desinstitucionalizar esses pacientes, reinseri-los na sociedade, promove-los como papel principal na construção de seu tratamento, ampará-los de maneira digna e holística ${ }^{2}$.

Após a Reforma Psiquiátrica, a identidade do enfermeiro começa a ser colocada em prática, fazendo com que o enfermeiro fosse capaz de estabelecer um vínculo com os pacientes e ampará-lo quanto suas necessidades patológicas, físicas e emocionais.

A mudança no papel do enfermeiro, compreende a assistência do paciente psiquiátrico de forma holística e reparadora. A partir dessas mudanças, faz-se necessário estudos para disponibilizar práticas assistenciais nas unidades psiquiátricas.

No ano de 2001, entrou em vigor a lei que promulgou a reforma psiquiátrica no Brasil, firmando ali uma responsabilidade de assistência capaz de oferecer assistência privilegiada de proteção e direitos dos pacientes com transtornos mentais ${ }^{2}$.

Sendo assim, a atuação do enfermeiro foi transformada, de maneira que o mesmo passa a ser crucial para essa inovação no tratamento psiquiátrico. O enfermeiro deixa de ser responsável pela sedação e contenção dos pacientes e passa a ser o responsável pela sua evolução, com intuito de promover interação e vínculo ${ }^{2,3}$.

A partir da Reforma Psiquiátrica, os pacientes necessitam ser inseridos nos mais diversos segmentos da saúde pública. Faz-se necessário que, independente da especialização do profissional enfermeiro, o mesmo tenha o manejo do cuidado psiquiátrico, capaz de assistir o paciente psiquiátrico de maneira integral e igualitária.

Atualmente, há um grande estigma acerca da referida assistência de enfermagem, inclusiva e integral, principalmente ao paciente portador de transtorno do espectro autista, por ser este um transtorno que dificulta $\mathrm{o}$ processo de interação assistencial e feedback do paciente e pelo fato da falta de abordagem da temática no campo da enfermagem.

Assim sendo, questiona-se a qualidade e o saber técnico da assistência de enfermagem ao paciente psiquiátrico, principalmente durante a infância, visto que é neste período da vida, onde ocorrem as principais descobertas e torna-se perceptível as principais características do ser humano, incluindo suas dificuldades, suas facilidades e seus objetivos, promovendo então, uma personalidade a este ser humano.

Sabe-se que a "infância é o período de crescimento que vai do nascimento à puberdade, ou seja, do zero aos doze anos de idade" ${ }^{4}$. E é nesta fase, em que muitas das vezes, a interação social e a verbalização é dificultosa por diversos fatores: falta de contato social, falta de diálogo com a criança e até mesmo, o autismo, que é do que se trata especificamente o estudo.

Trabalhar com o universo infantil, onde o lúdico é predominante, a verbalização é em muitos casos, escassa e os sentimentos são intensos, é uma missão com alto grau de dificuldade, ainda mais, tratando-se do universo infantil associado ao transtorno mental ${ }^{5}$.

Sendo assim, estabelece-se ao profissional enfermeiro, funções importantes no cuidado desses pacientes, incluindo o diagnóstico do espectro autista, diferenciando-se da não verbalização fisiológica, dada por falta de estímulos, por exemplo, além do acompanhamento e tratamento dessas crianças, inserindo-as na sociedade.

O objeto que norteia este estudo é: o cuidado de enfermagem a criança autista.

Diante da problemática apresentada, surge a seguinte questão norteadora: Qual o grau de conhecimento da equipe de enfermagem sobre cuidado prestado a criança autista?

A partir daí, traçamos os seguintes objetivos para o estudo: 1- Descrever o cuidado de enfermagem a criança autista; e 2 - Analisar o cuidado de enfermagem a criança autista.

\section{Material e métodos}

O presente estudo trata-se de uma revisão bibliográfica de literatura. Foi adotado para a execução deste presente estudo como estratégia metodológica, a revisão de literatura, através da revisão narrativa, que possibilita um maior contato com os relatos dos pesquisadores.

Foram utilizados artigos pesquisados publicados no ano de 2014 a 2019 nas bases de dados da literatura Latino-Americana e do Caribe em Ciências da Saúde (LILACS), do Scientific Electronic Library Online (Scielo) e Base de dados da Enfermagem (BDENF), coletados no período de agosto a outubro de 2019. Os descritores, para busca nas bases de dados, utilizados 
foram cuidados de enfermagem, transtorno do espectro autista, autismo, sendo identificados nos Descritores em Ciência da Saúde (DeCS). Os critérios de inclusão utilizados foram: a relação com autismo infantil; foram excluídos da pesquisa os artigos que mesmo abordando a temática solicitada, não exploravam a assistência de enfermagem à essa criança.

Com a busca dos dados foram encontrados inicialmente 1.278 artigos que envolviam a o tema de autismo, após aplicar os DECS, filtrou-se 8 artigos que abordavam o tema, no idioma Português e no período de 2014-2019. Após avaliação dos títulos, encontraramse o total de 6 artigos, após a leitura minuciosa dos artigos foi excluído o total de 3 artigos, resultando em somente 3 artigos que abordam a temática dos cuidados de enfermagem ao autismo infantil.

O instrumento para coleta de dados foi feito a partir de informações referentes aos artigos filtrados para esse estudo. Foram utilizadas as variáveis: ano em que foi publicado, tipo de estudo, autores, resultados, conclusão, país origem do estudo.

Os dados foram coletados e posteriormente, foram analisadas as informações neles inseridas. Após a análise, houve a compilação das mesmas. Em seguida, a análise descritiva foi realizada, a fim de compreender e expandir as informações sobre o tema escolhido, na finalidade de elaborar as referências teóricas.

\section{Resultados}

Para concretização do estudo, vimos a necessidade de demonstrar os artigos científicos dos quais utilizamos como fonte de análise desta pesquisa. Os mesmos encontram-se na tabela 1 .

$\mathrm{Na}$ relação de dados foi constatado que há um grande déficit de estudos científicos que abordem a temática.

Expressa-se uma necessidade de realizações de discussões que abordem a assistência de enfermagem à criança autista, visto que, o acervo bibliográfico referente ao tema é reduzido comparado as demais patologias psiquiátricas e o índice do TEA vêm crescendo em nosso país ${ }^{6}$.

Sabendo que sujeitos com TEA sofrem com preconceitos e estigmas sociais da doença, o que interfere de forma direta na prática de suas relações interpessoais e é nesta prática das relações interpessoais que o enfermeiro é capaz de colaborar positivamente, através de práticas como: diagnóstico precoce, acompanhamento do transtorno, olhar observador capaz de analisar o comportamento dessas crianças e mediante a análise auxiliá-los em suas dificuldades e nos desafios que encontram ${ }^{6}$.

Atualmente, perpetua-se uma fragilidade na assistência integral, universal e de equidade nos segmentos da saúde pública no Brasil, para com os autistas ${ }^{6}$. Isso se dá pelos diversos sinais e sintomas inclusos no transtorno do espectro autista, pela variabilidade etiológica e pela falta de apresentação do transtorno ainda nas academias de enfermagem ${ }^{6}$.

Pesquisas revelam que de um $n=15$, apenas 2 tinham conhecimento do transtorno e os demais, nunca tiveram contato sequer com a criança autista ${ }^{6}$. Sendo que, dois profissionais que asseguraram ter conhecimento sobre a temática, afirmaram ter preparo para assistir a criança portadora de TEA, mas negaram ter estruturas que colaborem com essa prática, tais como fluxogramas da assistência, anteriormente ${ }^{6}$.

Vê-se a necessidade de estudos científicos capazes de abordar o tema e fazê-los presentes desde a fase acadêmica até a fase de atuação do profissional enfermeiro, visto que, assim seria possível proporcionar um maior acalento, cuidado e melhor qualidade de vida a esses pacientes ${ }^{6}$.

Nota-se que os acadêmicos de enfermagem apresentam um nível de conhecimento mediano quanto ao transtorno do espectro autista, mas também notase que este conhecimento não partiu das instruções acadêmicas ${ }^{7}$.

Os acadêmicos entrevistados, afirmaram que seus rasos conhecimentos sobre o transtorno do espectro autista, foram adquiridos através das mídias sociais, tais como filmes e documentários ${ }^{6}$.

Sendo assim, percebe-se fragilidades para o reconhecimento do autismo, sendo elas: sintomatologia, devido a alta variabilidade dos sinais e sintomas ${ }^{6,7}$, o cuidado ético ao portador de TEA, baseado em evidencias nos diversos setores da saúde pública, devido ao déficit de estudos que abordem o tema nos acervos bibliográficos e a etiologia, devido à falta de abordagem nas academias?

Destaca-se ainda, o fato de que o profissional enfermeiro necessita ter o manejo da assistência a criança com TEA, capaz de planejar um cuidado abrangente, singular, que seja ainda, capaz de inserir os genitores e cuidadores, agindo junto à família, auxiliandoos no processo de compreensão das dificuldades apresentadas $^{6,7}$.

\section{Discussão}

São nos primeiros anos de vida, que os sintomas mais graves do TEA são diagnosticáveis. Os sintomas moderados são diagnosticáveis, na maioria das vezes, durante a fase escolar, em que a criança é inserida à prática do convívio social8. Visto que, não há um marcador biológico que seja capaz de diagnosticar o autismo, sendo assim, torna-se extremamente importante o acompanhamento do comportamento da criança, observando-a9. 
Tabela 1. publicações de artigos que abordam os descritores utilizados. Apresenta a descrição dos artigos selecionados de acordo com seus autores, ano de publicação, país de origem, objetivo, tipo de estudo e principais resultados no decorrer dos anos de 2015-2019.'

\begin{tabular}{|c|c|c|c|c|c|}
\hline AUTORES & ANO & PAÍS & OBJETIVO & $\begin{array}{l}\text { TIPO DE } \\
\text { ESTUDO }\end{array}$ & $\begin{array}{l}\text { RESULTADOS } \\
\text { OBTIDOS }\end{array}$ \\
\hline
\end{tabular}

Dartora, Denise

Dalmora; Mendieta,

Marjoriê da Costa;

Franchini, Beatriz.
Conhecer a percep- Pesquisa qualitativa, Observou-se que há ção da Equipe de descritiva e explora- incutido em cada Enfermagem frente tória. Os participantes profissional uma viao atendimento às do estudo foram seis são limitada sobre crianças autistas, na profissionais da equi- crianças autistas, por pediatria de um Hos- pe de enfermagem. vezes preconceituopital Universitário no A coleta de dados sa. O conhecimento Sul do Rio Grande do ocorreu em Janeiro empírico sobrepôs-se Sul.

de 2014, por meio de entrevista semiestruturada. A análise dos dados foi realizada a partir da análise temática de Minayo, a qual permitiu identificar três temas: visão dos profissionais de enfermagem sobre o autismo; equipe de Enfermagem: medo ou dúvida; fatores que interferem na assistência à criança autista.

Sena, Romeika Carla
Ferreira de; Reinal-
de, Elda Medeiros;
Silva, Glauber Weder
dos Santos; Sobreira,
Maura Vanessa Silva
2015 Brasil
Analisar a prática e
o conhecimento dos
enfermeiros da Estra-
tégia Saúde da Famí-
lia acerca do transtorno autístico.
Pesquisa explorató-

ria, com abordagem qualitativa, composta Utilizou-se a entrevista semiestruturada. A análise de dados deu-se através da análise representacional, aprovada pelo Comitê de Ética em Pesquisa da Universidade do Estado do Rio Grande do Norte (CEP/UERN $\mathrm{n}^{\mathrm{o}}$ 124/11).

Ferreira, Ana Caroline Souza Saraiva; Franzoi, Mariana André Honroato

2015 Brasil $\begin{aligned} & \text { Analisar o conheci- } \\ & \text { mento dos estudantes }\end{aligned}$

\section{Trat}

de Enfermagem de uma universidade púpor 15 enfermeiros.
Brasil

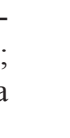
blica sobre os Transtornos do Espectro do Autismo (TEA). quantitativo, descri-
Trata-se de estudo tivo, realizado com 65 estudantes de graduação em Enfermagem. Coletaram-se os dados por meio de questionário, submetendo-os à análise estatística e apresentando-os em tabela. ao científico e com isso a assistência às crianças com autismo mostrou-se fragilizada.Considerações finais: a busca pelo conhecimento deve estar intrínseca em cada profissional, para assim, contribuir com uma assistência mais qualificada (AU)

Evidenciou-se insegurança e fragilidade no conhecimento dos enfermeiros sobre transtorno autístico em virtude de não terem conseguido definir autismo nem demonstrado vivência com pessoas autistas e relataram a inexistência de capacitações voltadas para o tema exposto

Evidenciaram-se os meios de comunicação como a principal fonte para a aquisição de informações sobre os TEA pelos estudantes. Apontaramse, como principais alterações dos TEA, as dificuldades nas interações sociais, o comprometimento na comunicação e o uso da linguagem verbal e não verbal, além de altas habilidades cognitivas. Verificouse que $90,8 \%$ não se sentem seguros para atender pessoas com TEA. 
Percebe-se que o diagnóstico precoce do TEA é de suma importância para que haja um planejamento da assistência desta criança, através de serviços médicos e serviços educativos. ${ }^{8}$

São evidenciadas muitas dificuldades por parte dos profissionais de saúde referente ao diagnóstico do transtorno do espectro autista ${ }^{6,7,10}$, parte delas são decorrente do fato de os profissionais não terem experiências com crianças portadoras de autismo suficiente para realizar determinado diagnóstico, fazendo com que o processo fique dificultoso ${ }^{10}$.

A singularidade da criança portadora de TEA é fator importante no diagnóstico precoce. Sua individualidade, aliada à grande variabilidade sintomatológica do autismo, muita das vezes, difere da sintomatologia citada nas bibliografias, o que dificulta também no diagnóstico precoce do autismo. ${ }^{6,10}$

Aliada a singularidade, a falta de vínculo com a criança e seus familiares, desconhecimento de seus hábitos e comportamentos, variações da sintomatologia, necessidade do diagnóstico individualizado, também são fatores que dificultam o processo de assistência à criança autista. ${ }^{10,11}$

Faz-se necessária um maior vínculo do profissional com o portador de TEA e os componentes de sua rede de cuidados, tais como: familiares, amigos e educadores, visto que, através da observação desses componentes é possível contribuir no conceito do comportamento de cada paciente, de forma individualizada, facilitando o processo de diagnóstico. ${ }^{10}$

Sendo assim, estabelece-se que as consultas prestadas às crianças portadoras de TEA, devem ser mais frequentes e prolongadas ${ }^{10}$, para conhecer mais os desafios que esta criança enfrenta, as suas necessidades de maneira holística e seus hábitos comportamentais.

Outro fator que dificulta a assistência de enfermagem à criança autista, é a falta de instrumentos eficazes para rastrear os sinais precoces do TEA em nosso país, influencia muito na dificuldade diagnóstica do transtorno. ${ }^{10,11}$ Nota-se então, que este fator é preocupante, uma vez que, através desses instrumentos muitas crianças podem ser tratadas previamente, evitando um alargamento das sintomatologias do transtorno. ${ }^{11}$

Sendo assim, é recomendado que estudos sejam realizados para que hajam instrumentos específicos para esse rastreio, a fim de evitar o diagnóstico tardio do transtorno, como se têm sido feito atualmente em nosso país ${ }^{11}$.

Com relação ao manejo de enfermagem, os enfermeiros têm papel fundamental na assistência, uma vez que, o profissional enfermeiro é capaz de desenvolver-se como um socializador, capaz de compreender a criança, reconhecer seus limites e orientar sua rede de apoio e família. ${ }^{12}$

O enfermeiro é capaz de prestar uma assistência humanizada à criança e sua família, capaz de promover melhor qualidade de vida à criança portadora de TEA. Uma vez que é através da enfermagem, que a observação do comportamento é realizada. Através da enfermagem, também são criados os vínculos entre os segmentos da saúde pública e a criança e seus familiares. ${ }^{12}$

Evidencia-se então que as dificuldades encontradas pela equipe de enfermagem acerca do manejo da assistência à criança portadora de TEA ${ }^{13}$.

O manejo da enfermagem na assistência à criança autista, deve compreender que a criança pertence a um determinado grupo responsável por seu cuidado, e este cuidado requer paciência e destreza. ${ }^{12,13 .}$

Deve-se ressaltar ainda, que o fato de a criança autista ter sua dependência por cuidadores aumentada, pode ser um fator estressante para a família. Assim sendo, estima-se que o manejo do profissional enfermeiro vise a criança e sua família, capaz de promover uma assistência eficaz, evitando o adoecimento desse cuidador. ${ }^{13}$

Em contrapartida, este manejo profissional só será alcançado quando as dificuldades como falta de conhecimento sobre o transtorno forem supridas. ${ }^{11,13}$

Essas dificuldades poderiam ser supridas com o aumento de literaturas, estudos científicos que abordem o tema e maior diversidade nos acervos bibliográficos que relatem experiências com crianças portadoras de TEA, além da inserção de teorias e práticas sobre o manejo da assistência à criança com TEA, durante a academia. ${ }^{6,7,13}$

Para que a assistência eficaz do profissional enfermeiro à criança portadora de TEA seja colocada em prática, é necessário que haja empatia. O profissional deve disponibilizar-se a ser posto no lugar do outro, aceitando-o e aprendendo com o mesmo. ${ }^{13}$

O déficit de estudos que abordam o tema dificulta grandemente a assistência de enfermagem. Isso se dá, pelo fato dos profissionais enfermeiros não darem a devida importância à saúde mental, fazendo com que não haja estudos suficientes na área. ${ }^{14}$

Percebe-se que para que haja uma assistência de enfermagem capaz de diagnosticar o transtorno, acompanhar o desenvolvimento e promover uma melhor qualidade de vida, é necessário estudo bibliográfico capaz de orientar, complementar o conhecimento e auxiliar na prática assistencial da enfermagem no campo do tratamento do autismo. ${ }^{12,13}$

\section{Conclusão}

$\mathrm{O}$ estudo buscou analisar o cuidado de enfermagem prestado à criança com autismo. A partir da análise, foi visto que encontra-se defasado o conhecimento acerca do transtorno autístico dentre os profissionais enfermeiros. A falta de discussão sobre o assunto a começar nos campi universitários, fazem 
com que este o TEA seja conhecido, muito das vezes, somente pelos profissionais psiquiátricos, que atuam na área de saúde mental.

Isso reflete em nossa sociedade de maneira alarmante, ao percebermos a falta de habilidade dos profissionais de enfermagem na assistência às crianças portadoras de TEA.

Mediante o que foi minuciosamente estudado, vemos a necessidade da inserção de disciplinas nas grades universitárias, que abordem práticas alternativas de assistência de enfermagem, para que o profissional enfermeiro possa teruma capacidade de percepção ampla, capaz de avaliar os sinais de TEA precocemente.

A assistência ao paciente psiquiátrico não pode ser eleita uma prática restrita ao profissional enfermeiro psiquiátrico, visto que, os pacientes psiquiátricos circundam por todas as áreas do sistema de saúde, desde atenção primária à terciária.

Visto isso, faz-se necessária a capacitação dos profissionais enfermeiros, para que estejam aptos para prestar uma assistência otimizada e especifica, capaz de proporcionar inserção, de estabelecer um vínculo e promover uma verbalização, ainda que, mínima. ${ }^{15}$

Sendo assim, após as melhorias necessárias, a assistência do profissional enfermeiro poderá ser classificada como eficaz e cumpridora de todos os princípios básicos do SUS.

\section{Referências}

1. Stockinger RC. Reforma psiquiátrica brasileira: perspectivas humanistas e existenciais. Editora Vozes, 2007.

2. Saúde Brasil 2005 - BVS MS - Ministério da Saúde, acessado em 25/08/19, às 14:20 horário de Brasília.

3. Almeida Filho AJ, Moraes AEC, Almeida PMA. Atuação do enfermeiro nos centros de atenção psicossocial: implicações históricas da enfermagem psiquiátrica. Revista da Rede de Enfermagem do Nordeste, 2009;10(2):158-65.

4. FSS, Fundo Social de Solidariedade et al. Estatuto da Criança e do Adolescente. 1990.

5. Souza D. Equivalência de estímulos e autismo: Uma revisão de estudos empíricos. Psicologia: Teoria e Pesquisa, 2010.

6. Sena RCF et al. Prática e conhecimento dos enfermeiros sobre o autismo infantil. Revista de Pesquisa Cuidado é Fundamental Online, 2015;7(3):2707-16.

7. Ferreira ACSS, Franzoi MAH. Conhecimento de estudantes de enfermagem sobre os transtornos autísticos. Rev. enferm. UFPE on line, 2019:51-60

8. Albores-Gallo, L. et al. Dificultades en la evaluación y diagnóstico del autismo: Una discusión. Salud mental, 2008;31(1):37-44.

9. Costa MF, Nunesmaia HGS. Diagnóstico genético e clínico do autismo infantil. Arquivos de Neuropsiquiatria, 1998; 56(1), 24-31. Recuperado em 10 de março de 2012, de http://www.scielo.br

10. Oliveira JC. Dificuldades encontradas pelos profissionais da saúde ao realizar diagnóstico precoce de autismo. Psicologia argumento, 2017;32(77).

11. Seize MM, Borsa JC. Instrumentos para rastreamento de sinais precoces do autismo: revisão sistemática. Psico-USF, 2017;22(1):161-76.
12. Carniel EL, Saldanha LB, Fensterseifer LM. A atuação do enfermeiro frente à criança autista. Pediatria. Säo Paulo, 2010:255-60.

13. Dartora DD, Mendieta MC, Franchini B. A equipe de enfermagem e as crianças autistas. Journal of Nursing and Health, 2014;4(1):27-38.

14. Marques IBN, Alcântara FL, Alves M, Casanova EG. O Cuidar em Saúde Mental na Atenção Básica Sob a Lógica dos Enfermeiros. Revista Pró-univerSUS, Vassouras, 2011 jul./dez.;2(2):53-62.

15. Ribas LB. O Cuidado de Enfermagem a criança com transtorno do espectro autista: um desafio no cotidiano. [Monografia]. Vassouras (RJ): Universidade de Vassouras; 2019. 2017. 